\title{
Improved Response Time Analysis of Tasks Scheduled under Preemptive Round-Robin
}

\author{
Razvan Racu, Li Li, Rafik Henia, Arne Hamann, Rolf Ernst \\ Institute of Computer and Communication Network Engineering \\ Technical University of Braunschweig, Germany \\ \{racu|lili|henia|hamann|ernst\}@ida.ing.tu-bs.de
}

\begin{abstract}
Round-Robin scheduling is the most popular time triggered scheduling policy, and has been widely used in communication networks for the last decades. It is an efficient scheduling technique for integration of unrelated system parts, but the worst-case timing depends on the system properties in a very complex way. The existing works on response time analysis of task scheduled under RoundRobin determine very pessimistic response time bounds, without considering in detail the interactions between tasks. This may lead to a degradation of the efficiency of Round-Robin scheduling algorithm, and becomes a practical obstacle to its application in realtime systems. In this paper we present an approach to compute much tighter best-case and worst-case response time bounds of tasks scheduled under preemptive Round-Robin, including also the effects of the scheduling algorithm.
\end{abstract}

\section{Categories and Subject Descriptors}

B.8 [Performance and Reliability]: Performance Analysis and Design Aids; C.3 [Special-Purpose and Application-Based Systems]: Real-Time and Embedded Systems

\section{General Terms}

Design, Performance, Reliability, Verification

\section{INTRODUCTION}

Round-Robin scheduling is known as a very effective time triggered scheduling policy. It allows an easy balance of resource utilization between tasks that are ready to be executed. An example is the POSIX standard. In real-time systems Round-Robin scheduling can be used as a best effort strategy. Moreover, in high load situations, it still guarantees a minimum service rate for each task, as a minimum performance guarantee. This makes it a good strategy for systems integration. For instance, weighted Round-Robin is the preferred scheduling strategy used in packet-based communication systems [1]. Recently, such systems has been used together with different real-time applications [4], which requires to guarantee the satisfiability of the imposed constraints.

If the deadlines in real-time systems are firm or hard, worst-case load situations must be assumed. This usually means that only the time slot assigned to a task is counted, resulting in the same performance as static time-driven scheduling, i.e. TDMA. In practice,

Permission to make digital or hard copies of all or part of this work for personal or classroom use is granted without fee provided that copies are not made or distributed for profit or commercial advantage and that copies bear this notice and the full citation on the first page. To copy otherwise, to republish, to post on servers or to redistribute to lists, requires prior specific permission and/or a fee.

CODES+ISSS'07, September 30-October 3, 2007, Salzburg, Austria.

Copyright 2007 ACM 978-1-59593-824-4/07/0009 ...\$5.00. we often find that TDMA is preferred in such cases due to its simpler control and predictable behavior. Examples are the automotive FlexRay [2] or the TTP protocols [8].

However, the pessimistic assumption is unnecessary if task properties can be taken into account, leading to a much better proven worst-case timing compared to TDMA. The corresponding schedulability analysis is, however, far more complicated. This is due to the dynamic assignment of the time slots, that depends on both the time slot order, and the activation and execution times of all other tasks sharing the resource.

There is a host of work on efficient and fair implementations of Round-Robin schedulers, most of them for communication networks $[13,16,5,7]$. However, these works do not consider the real-time aspects of the scheduling, and if they do so, they only determine very conservative latency bounds.

Later on, several network analysis methods provide more insight into the real-time performance of networks. Some of them use probabilistic models to describe the workload arrival or the communication times of the connections [18, 20,21], which is not adequate for the analysis of hard real-time systems.

Raha et al. [14] use a deterministic model for traffic description, but the computed response time bounds are still very conservative, since the analysis does not capture in detail the interaction between the communications sharing the same resource.

Migge et al. [12] use a mathematical model based on trajectories [11] to compute response time bounds for deterministic task models scheduled under Round-Robin within fixed-priorities.

In this paper, we present an approach to preemptive Round-Robin scheduling analysis for a set of tasks characterized by arrival functions, including burst behavior. The approach uses an event model interface to allow the analysis of tasks with deterministic workload bounds.

The rest of the paper is structured as follows: Section 2 presents the computational model with details about the workload arrival model and about the Round-Robin scheduler. Section 3 describes the worst-case execution scenario of a task and presents the algorithm to compute the worst-case response time. The algorithm flow is explained using a simple task set example. Thereafter, Section 4 presents a best-case response time algorithm based on a conservative approximation of the scenario leading to the shortest task response time. Section 5 considers the effects of the scheduling algorithm on the worst-case response time.

\section{COMPUTATIONAL MODEL}

\subsection{Task set}

In this paper we address the problem of schedulability analysis for a set of $n$ tasks $\mathcal{T}=\left\{\tau_{1}, \tau_{2}, \ldots, \tau_{n}\right\}$, executed on a processing resource using Round-Robin arbitration strategy. The tasks are fully preemptive, i.e. their execution can be interrupted at any point in time. The tasks are assumed to be independent, i.e. no blocking occurs between tasks, and no correlation exists between the release patterns of the tasks. 
The tasks are activated by activating events, generated in a multitude of ways, including expiration of a timer, external or internal interrupt, availability of data for processing, etc. The timing behavior of the activating events is described in detail in Section 2.2.

At each activation a task requires the resource for a bounded period of time, called task execution time. The minimum bound of the execution time of a task $\tau_{i}$ is called best-case execution time, denoted $C_{i}^{\text {min }}$. Analogously, the maximum bound is called worstcase execution time and is referred to as $C_{i}^{\max }$.

Successive task activations are allowed to overlap each other. In order to prove the schedulability of the task set, one must compute the response times of the tasks and compare them against a set of predefined deadlines. There is no restriction with respect to task deadlines. This assumption shows the generality of the response time analysis presented in this paper.

\subsection{Event models}

The activating events of a task are captured by an event stream. The behavior of an event streams is described using event models.

The classical event models used in the scheduling theory use a set of parameters to describe the timing of the events within an event stream. Hence, a periodic event model is characterized by the period $(\mathcal{P})$ [9], a periodic with jitter event model [19] is described by two parameters, the period $(\mathcal{P})$ and the jitter $(\mathcal{J})$ and a sporadic event model [17] is described by the minimum inter-arrival distance $\left(d^{-}\right)$.

For the purpose of the response time analysis, the event models are mathematically represented using a set of functions: $\eta^{+}(\Delta t)$, $\eta^{-}(\Delta t), \delta^{-}(n)$ and $\delta^{+}(n)$ [15]. The $\eta$ functions are called arrival functions and describe the maximum and minimum number of events arriving in the interval $\Delta t$. The $\delta(n)$ functions are called distance functions and describe the minimum and maximum distance between any event $i$ and the $(n-1)$-th event after $i$ in the corresponding event stream [15].

In this paper, we use the four functions to capture the workload of the tasks in the response time equations.

\subsection{Round-Robin scheduler}

The Round-Robin (RR) scheduler assigns the resource to each task in a cyclic fashion. Each task $\tau_{i}$ has assigned a predefined amount of time, called time slot, where it is granted the resource. For brevity, we denote the time slot of $\tau_{i}$ by $\theta_{i}$. The sum of all time slots is called RR-turn. The order in which the time slots are executed within a RR-turn is predefined. We assume the time slots are ordered according to the corresponding task indices. After $\theta_{n}$ a new RR-turn starts. During each turn every task accesses the resource only within its time slot.

If no instance of $\tau_{i}$ is pending at the beginning of $\theta_{i}$, then no processor time is assigned to task $\tau_{i}$ and the scheduler examines $\tau_{i+1}$. If at least one activation of $\tau_{i}$ is waiting for execution at the beginning of $\theta_{i}$, then $\tau_{i}$ is allowed to execute without interruption for at most $\theta_{i}$ units of time. If the task did not complete its execution at the end of its time slot, it is interrupted (preempted) by the scheduler. In case that $\tau_{i}$ completes its execution before its time slot expires, the next pending activations of $\tau_{i}$, are executed for the time left in the current time slot. If the time slot did not expire and there are no more waiting task instances, the scheduler switches to the task corresponding to the next slot in the RR-turn.

\section{WORST-CASE RESPONSE TIME}

\subsection{Critical instant}

In order to find the worst-case response time, one must determine the scheduling scenario when the longest response time occurs. Liu and Layland [10] defined the critical instant of a task to be the instant at which a request for that task will have the largest response time. Lehoczky [9] proved that the critical instant of a task $\tau_{i}$ on a fixed-priority scheduler, occurs at the beginning of the level-i busy period. A level-i busy period is defined as the maximum time for which a processor executes tasks with priority higher than or equal to the priority of $\tau_{i}$. However, under Round-Robin, the task priorities dynamically change in time, and therefore, the definition of the level-i busy period must be first adapted and then applied for the computation of the worst-case response time.

In general, the maximum busy period is the maximum time for which a processor is busy executing tasks. Intuitively, the critical instant of a task under Round-Robin is the instant when the task has the lowest priority among all other tasks. This scenario guarantees the longest waiting time until the task may access the resource again.

LEMMA 1 (MAXIMUM BUSY PERIOD). The maximum busy period occurs when all tasks mapped on the resource are simultaneously released and request the worst-case execution time. Additionally, all subsequent task activations arrive as soon as possible (worst-case load condition).

Proof. As in [10, 6, 9].

THEOREM 1 (CRITICAL INSTANT UNDER ROUND-ROBIN). The critical instant of a task under Round-Robin occurs at the beginning of the maximum busy period instantiated right after the time slot of the analyzed task expired.

Proof. Let $\tau_{n}$ be the analyzed task and $t_{0}$ the time instant when the time slot $\theta_{n}$ expires. The beginning of the next $\theta_{n}$ occurs at:

$$
t=t_{0}+\sum_{j=1}^{n-1} \min \left(\theta_{j}, w_{j}\right)
$$

where $w_{j}$ represents the maximum execution time requested by task $\tau_{j}$ up to the start time of its slot, denoted as $t_{(j)}$. Using the standard event model interface described in Section 2.2, $w_{j}$ can be calculated as

$$
w_{j}=C_{j}^{\max } \cdot \eta_{j}^{+}\left(t_{(j)}\right) \text { where } t_{(j)}=\sum_{k=1}^{j-1} w_{k}
$$

Assume that $\tau_{n}$ is released with an offset $\phi_{n}$ after $t_{0}$. Since $\tau_{n}$ can not start the execution earlier than $t$, the response time of $\tau_{n}$ will be $\phi_{n}$ units of time shorter. Obviously, the longest response time occurs for $\phi_{n}=0$.

If $\tau_{n}$ is released before $t_{0}$, then $\tau_{n}$ gets the chance to start its execution in the slot finishing at $t_{0}$, and thus, its waiting time may be shorter than the case when $\tau_{n}$ starts at $t_{0}$. Hence, $t_{0}$ represents the critical instant.

\subsection{Worst-case response time algorithm}

Consider a task set consisting of four preemptive tasks, $T 1, T 2$, $T 3$ and $T 4$ executed on a Round-Robin resource. $T 1, T 2, T 3$ are periodically activated. Task $T 4$ experiences a burst activation due to a large jitter. The distance between the burst events is controlled by the minimum distance parameter $\left(d^{-}\right)$of the input event model. After the burst events expired, the task is released periodically, as it can be seen in Figure 1.

Table 1 shows the parameters of the input event models, the worst-case execution times, and the time slot values corresponding to the task set. The time slots are scheduled in the following order: $\theta_{1} \rightarrow \theta_{2} \rightarrow \theta_{3} \rightarrow \theta_{4}$.

\begin{tabular}{|c|c|c|c|c|c|}
\hline \multirow{2}{*}{$\begin{array}{l}\text { Task } \\
\left(\tau_{i}\right)\end{array}$} & \multicolumn{3}{|c|}{ Activation model } & \multirow{2}{*}{$\begin{array}{l}\text { Execution time } \\
\left(C_{i}^{\max }\right)\end{array}$} & \multirow{2}{*}{$\begin{array}{c}\text { Time slot } \\
\theta_{i}\end{array}$} \\
\hline & $\mathcal{P}_{i}$ & $\mathcal{J}_{i}$ & $d^{-}$ & & \\
\hline$\overline{T 1}$ & 15 & - & - & 3 & 2 \\
\hline$T 2$ & 50 & - & - & 10 & 3 \\
\hline$T 3$ & 30 & - & - & 7 & 5 \\
\hline$T 4$ & 20 & 50 & 5 & 5 & 7 \\
\hline
\end{tabular}

Table 1: The task parameters

The algorithm to determine the worst-case response time of a task under Round-Robin uses the windowing technique proposed 
by Lehoczky [9], and refined latter by Tindell [19]. The approach finds the worst-case response time of a task by checking different time windows within the busy period. The $q$-window of task $\tau_{i}$, denoted also $w_{i}(q)$, represents the time interval between the instant when the maximum busy period was initiated $\left(t_{0}\right)$ and the time instant when the $q$-th activation of $t_{i}$ completes its execution. In other words, the $q$-window of $\tau_{i}$ represents the response time of the first $q$ activations of $\tau_{i}$ in the maximum busy period.

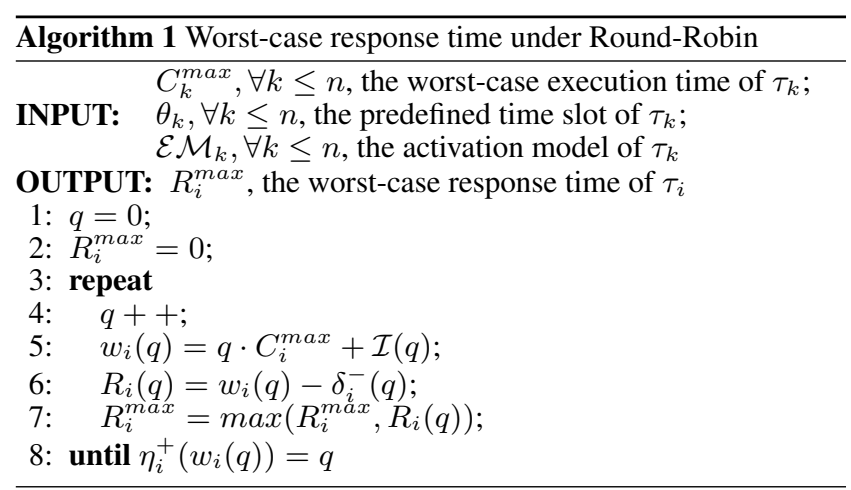

Algorithm 1 computes the worst-case response time of $\tau_{i}$. During each loop iteration the $q$-th window of $\tau_{i}$ is analyzed. The value of $w_{i}(q)$ (line 5) is equal to the execution demand of the first $q$ activations of $\tau_{i}, q \cdot C_{i}$, plus the interference, $\mathcal{I}(q)$, with other tasks mapped on the same resource. $\mathcal{I}(q)$ is explained in detail later in this section.

$\delta_{i}^{-}(q)$ returns the arrival time of the $q$-th activation of $\tau_{i}$. Line 7 returns the response time of the $q$-th activation of $\tau_{i}$ within the busy window. The maximum over all $R_{i}(q)$ represents the worstcase response time $R_{i}^{\max }$ of $\tau_{i}$.

The loop terminates when the number of activations released in the $q$-th window does not exceed $q$, i.e. the first $q$ activations of $\tau_{i}$ have been completely processed before the $(q+1)$-th activation arrived. The maximum number of activations that arrive in the time window $w_{i}(q)$ is computed using the upper-bound arrival function, $\eta_{i}^{+}$.

Notice that both functions, $\delta_{i}^{-}$and $\eta_{i}^{+}$are obtained from the activation model $\mathcal{E} \mathcal{M}_{i}$ of $\tau_{i}$.

Figure 1 shows the worst-case scheduling scenario of task $T 4$ in the example introduced above. The worst-case response time algorithm starts with $q=1$ and calculates the value of the 1st window of $T 4$. As we can observe in the figure, $w_{4}(1)=15$, and, since first activation of $T 4$ was released at $t_{0}=0$, the response time of this activation is also 15 . Moreover, we observe that the 2 nd activation of $T 4$ was released within the 1st window, and thus, the algorithm investigates also the 2 nd window. The value of $w_{4}(2)$ is equal to 27 and, because the 2 nd activation of $T 4$ was released at time instant 5 , its response time is 22 . The algorithm continues until $q=7$. We observe that the 8th activation of $T 4$ is released after the 7 th activation has completed the execution, and therefore the algorithm terminates. The worst-case response time of $T 4$ occurs for the $3 \mathrm{rd}$ and 4 th activations of the task.

The interference $\mathcal{I}(q)$ of the analyzed task with other tasks, during the $q$-th window is computed as:

$$
\mathcal{I}(q)=\sum_{k=1}^{K(q)} \mathcal{I}_{k}, \text { where } K(q)=\left\lceil\frac{q \cdot C_{i}^{\max }}{\theta_{i}}\right\rceil
$$

where $\mathcal{I}_{k}$ represents the interference with other tasks during the $k$ th RR-turn, and $K(q)$ is the total number of RR-turns required by $q$ activations of $\tau_{i}$ to complete. In other words, $\mathcal{I}_{k}$ represents the total time that all other tasks used the processor during turn $k$.

For example, in Figure 1, the time interval $[31,41]$ corresponds to $\mathcal{I}_{3}$, and represents the time tasks $T 1, T 2$ and $T 3$ used the processor in the third turn. Every $\mathcal{I}_{k}$ is defined as $\mathcal{I}_{k}=\sum_{j=1}^{n-1} \mathcal{I}_{k, j}$, where $\mathcal{I}_{k, j}$ is the interference in the $k$-th RR-turn with the task whose predefined time slot order in the RR-turn is equal to $j$, assuming that the time slot of the analyzed task has the predefined time slot order $n$.

If we refer again to Figure 1 , the time interval $[22,24]$ corresponds to $\mathcal{I}_{2,3}$ and represents the time spent by $T 3$ in the second turn.

The interference $\mathcal{I}_{k, j}$ is determined by the remaining execution demand of $\tau_{j}$ at the beginning of $\theta_{j}$ in the $k$-th turn.

Since the remaining demand might not necessarily use up the entire time slot $\theta_{j}$, one must iteratively check if new activations of $\tau_{j}$ that arrived during the consumed time of $\theta_{j}$, can be processed in the available time in $\theta_{j}$. Therefore, $\mathcal{I}_{k, j}$ is described as follows:

$$
\mathcal{I}_{k, j}=\sum_{r=1}^{R} \operatorname{pad}_{k, j}(r), R \text { such that } \operatorname{pad}_{k, j}(r) \neq 0
$$

A pad represents the time spent in a time slot by all unserved task activations already arrived before the start time of the pad.

For example, if a slot contains multiple pads, the first pad in the slot corresponds to the execution of the task activation arrived before the slot started and not served in the previous slots. The value of the second pad corresponds to the execution of the task activations arrived during the execution of the first pad, and so on. It is very important to notice that the value of a pad can not be larger than the unused time in the corresponding slot.

The value of $R$ in Equation 4 defines the maximum number of pads in a slot. The pads in a slot are indexed over $r . \operatorname{pad}_{k, j}(r)$ represents the $r$-th pad in time slot $\theta_{j}$ of the $k$-th turn.

In Figure 1, the only slot with multiple pads is the slot of $T 2$ in the fourth RR-turn: one is used to finish the first activation of $T 2$ and the other one is used to execute a part of the second activation of $T 2$, which arrived during de execution of $\operatorname{pad}_{4,2}(1)$.

The value of a pad in time slot $\theta_{j}$ is given by the remaining execution demand of $\tau_{j}$ at the beginning of the pad and the free amount of time in $\theta_{j}$.

$$
\begin{aligned}
\operatorname{pad}_{k, j}(r)= & \min \left(l_{k, j}(r), C_{j}^{\text {max }} \cdot \eta_{j}^{+}\left(t_{k, j}+\sum_{s=1}^{r-1} p a d_{k, j}(s)\right)\right. \\
& \left.-\sum_{p=1}^{k-1} \mathcal{I}_{p, j}-\sum_{s=1}^{r-1} p a d_{k, j}(s)\right)
\end{aligned}
$$

The first term in the min function in Equation 5 represents the unused time in time slot $\theta_{j}$ at the beginning of $\operatorname{pad}_{k, j}(r)$. This time is calculated as follows:

$$
l_{k, j}(r)=\theta_{j}-\sum_{s=1}^{r-1} \operatorname{pad}_{k, j}(s)
$$

The second term in the min function represents the remaining execution demand of $\tau_{j}$ at the beginning of $\operatorname{pad}_{k, j}(r) . \eta_{j}^{+}$returns the total number of activations of $\tau_{j}$ since $t_{0} . t_{k, j}$ represents the starting time of $\theta_{j}$ relative to $t_{0}$, in the $k$-th RR-turn.

$$
t_{k, j}=\sum_{p=1}^{k-1} \mathcal{I}_{p}+(k-1) \theta_{i}+\sum_{u=1}^{j-1} \mathcal{I}_{k, u}
$$

The sum of the first and second terms in Equation 7 represents the value of the first $(k-1)$ RR-turns. The last term represents the time consumed in the $k$-th RR-turn by tasks with time slot order higher than $\tau_{j}$.

In Figure 1, the value of $t_{5,3}$ is calculated as the interference in the first four RR-turns, plus the time $T 4$ has been executed in the first four turns, plus the execution of $T 1$ and $T 2$ in the fifth turn.

The value of $\operatorname{pad}_{k, j}(r)$ in Equation 5 depends on the values of 


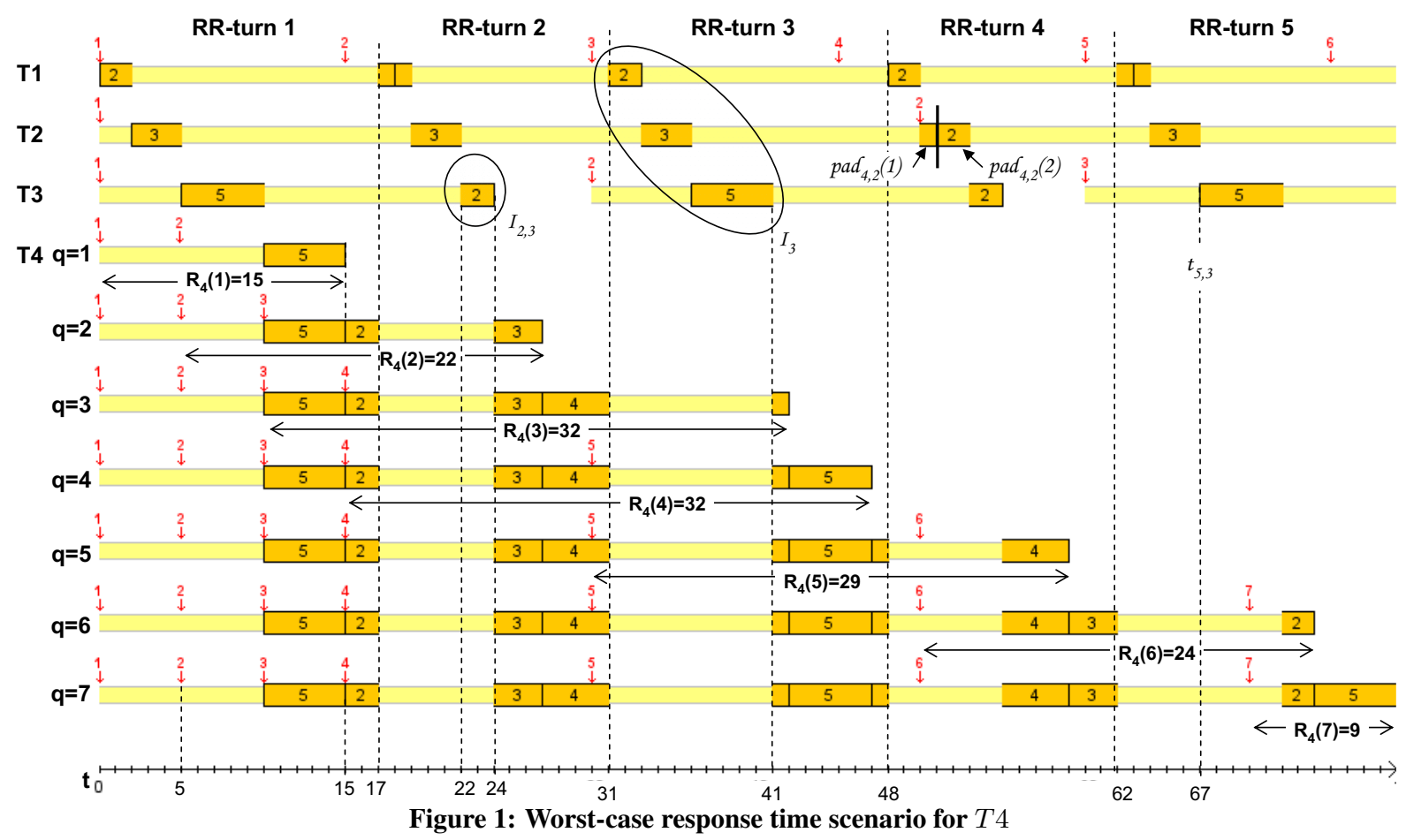

all $\operatorname{pad}_{k, j}(s)$, where $s<r$. $\operatorname{pad}_{k, j}(1)$ is calculated as follows:

$$
\operatorname{pad}_{k, j}(1)= \begin{cases}\theta_{j} & E_{k, j} \geq \theta_{j} \\ E_{k, j} & E_{k, j}<\theta_{j}\end{cases}
$$

$E_{k, j}$ in Equation 8 represents the remaining execution demand of task $\tau_{j}$ at the beginning of $\theta_{j}$, in the $k$-th RR-turn.

$$
E_{k, j}=C_{j}^{\max } \cdot \eta_{j}^{+}\left(t_{k, j}\right)-\sum_{p=1}^{k-1} \mathcal{I}_{p, j}
$$

The values of $\operatorname{pad}_{k, j}$ belonging to $\theta_{j}$ are iteratively calculated until first $p a d_{j, k}$ value equal to zero is found. In Equation 5 this means that either the time slot $\theta_{j}$ has been entirely used up, or the execution demand of $\tau_{j}$ has been completely processed. The value of $R$ in Equation 4 represents the total number of pads iteratively calculated using Equation 5.

Table 2 shows the worst-case response times of the task set, computed using the Algorithm 1 and the algorithms proposed by Raha [14] and Migge [12]. Tasks $T 1$ and $T 2$ are unschedulable according to Raha's algorithm. This is because the load of each task is larger than the service rate assigned to these tasks. Notice that, the response times computed by Algorithm 1 and the approach proposed in [12] are identic. However, in our opinion, the analysis in [12] suffers from the complex computational model.

Table 2: Computed worst-case response times

\begin{tabular}{|c|c|c|c|}
\hline & Algorithm 1 & Raha [14] & Migge [12] \\
\hline \hline T1 & 46 & unschedulable & 46 \\
T2 & 60 & unschedulable & 60 \\
T3 & 31 & 31 & 31 \\
T4 & 32 & 35 & 32 \\
\hline
\end{tabular}

\section{BEST-CASE RESPONSE TIME}

\subsection{The least critical instant}

The best-case response time analysis computes the shortest response time of a task. Intuitively, in order to experience the shortest response time, the task must be released at the instant when it has the highest priority among all other tasks. This occurs whenever the task is released at the beginning of its time slot.

Opposite to the concept of maximum busy period, one can define the maximum idle period with respect to a given set of tasks, as the maximum time for which the resource does not process any task in the given set.

THEOREM 2 (BEST-CASE RESPONSE TIME). The best-case response time of a task $\tau_{i}$ under Round-Robin scheduling occurs when the task is released at the beginning of the maximum idle period of all other tasks mapped on the same resource as $\tau_{i}$.

PRoOF. Let $\mathcal{G}=\mathcal{T}-\left\{\tau_{i}\right\}$ be the set of tasks executed on the same resource as $\tau_{i}$, and let $t_{0}$ be the time instant indicating the beginning of the maximum idle period of all tasks in $\mathcal{G}$. If $\tau_{i}$ is released before $t_{0}$, then at least one task in $\mathcal{G}$ is active and interferes with $\tau_{i}$.

If $\tau_{i}$ is released after $t_{0}$, then the interference of $\tau_{i}$ with the next activations of the tasks in $\mathcal{G}$ occurs earlier, and may extend the response time of $\tau_{i}$.

Contrary to maximum busy period, the maximum idle period makes assumptions about the completion of the tasks and not about task activation. Moreover, the dynamic behavior of the priority function in Round-Robin scheduling makes difficult to find out a relation between the task activation scenario leading to the maximum idle period.

Instead, we use a hypothetical scenario similar to [3] that leads to a conservative maximum idle time. We use this scenario to construct the least critical instant of a task $\tau_{i}$ scheduled under Round-Robin:

1. Task $\tau_{i}$ is released at an instant $t_{0}$.

2. Each task $\tau_{j}, j \neq i$ is released $C_{j}^{\text {min }}$ time units before $t_{0}$, executes without interruption and completes its execution at $t_{0}$. The next activations of the tasks arrive as late as possible.

The second statement represents the best-case load condition and ensures that the interference of $\tau_{i}$ with other tasks occurs as late as possible. Obviously, such a scenario is not possible in practice, as all tasks must use the resource simultaneously before $t_{0}$, in order to finish at the same time. 
The first statement guarantees that $\tau_{i}$ gets executed immediately after its activation, since at $t_{0}$ no other task except $\tau_{i}$ is waiting for execution.

Figure 2 shows the hypothetical scenario, where $t_{0}$ indicates the least critical instant.

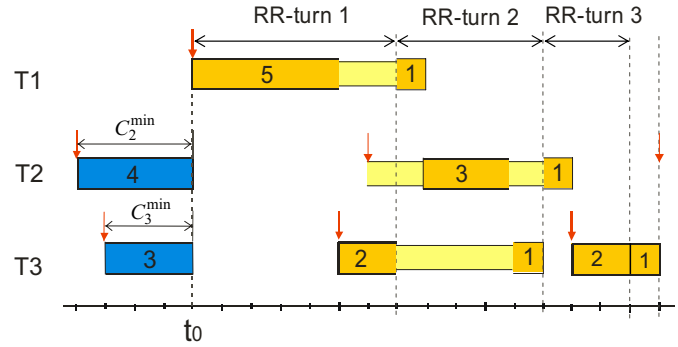

Figure 2: The least critical instant under RR

\subsection{Best-case response time algorithm}

In order to find the shortest response time of $\tau_{i}$ only the execution of the first activation of $\tau_{i}$ must be analyzed. The best-case response time is

$$
R_{i}^{\text {min }}=C_{i}^{\text {min }}+\mathcal{I}^{\text {min }}
$$

where $C_{i}^{\text {min }}$ represents the minimum execution time of $\tau_{i}$. The interference $\mathcal{I}^{\text {min }}$ of $\tau_{i}$ with other tasks is defined as

$$
\mathcal{I}^{\text {min }}=\sum_{k=1}^{K^{\prime}} \mathcal{I}_{k}, \text { where } K^{\prime}=\left\lceil\frac{C_{i}^{\text {min }}}{\theta_{i}}\right\rceil-1
$$

Since $\tau_{i}$ is activated at the beginning of its time slot, there is no interference in the last RR-turn. Therefore, only the interference in the first $\left\lceil\frac{C_{i}^{\min }}{\theta_{i}}\right\rceil-1$ turns must be calculated.

The interference $\mathcal{I}_{k}$ in the $k$-th RR-turn is defined as $\mathcal{I}_{k}=$ $\sum_{j=2}^{n} \mathcal{I}_{k, j}$ where $\mathcal{I}_{k, j}$ is the interference in the $k$-th RR-turn with the task whose predefined time slot order is equal to $j$, assuming that the time slot order of the analyzed task is 1 .

$\mathcal{I}_{k, j}$ is computed using Equation 4. The values of $\operatorname{pad}_{k, j}$ in Equation 4 are calculated as follows:

$$
\begin{aligned}
\operatorname{pad}_{k, j}(r)= & \min \left(l_{k, j}(r), C_{j}^{\text {min }} \cdot \eta_{j}^{-}\left(t_{k, j}+C_{j}^{\text {min }}+\right.\right. \\
& \left.\left.\sum_{s=1}^{r-1} \operatorname{pad}_{k, j}(s)\right)-\sum_{p=1}^{k-1} \mathcal{I}_{p, j}-\sum_{s=1}^{r-1} \operatorname{pad}_{k, j}(s)\right)
\end{aligned}
$$

The $\eta_{j}^{-}$function in above equation returns the total number of activations of task $\tau_{j}$ arrived until the beginning of $\operatorname{pad}_{k, j}(r)$.

The unused time in the time slot $\theta_{j}$ at the end of $p a d_{k, j}(r-1)$ is computed using Equation 6. The time at the beginning of $\theta_{j}$ in the $k$-th turn is

$$
t_{k, j}=\sum_{p=1}^{k-1} \mathcal{I}_{p}+k \cdot \theta_{i}+\sum_{u=2}^{j-1} \mathcal{I}_{k, u}
$$

Notice that, contrary to worst-case scenario, $t_{k, j}$ contains also the execution of $\tau_{i}$ in the $k$-th turn, as $\theta_{i}$ is the first time slot scheduled in the RR-turn.

The first value of $\operatorname{pad}_{k, j}$, is calculated using Equation 8 in Section 3.2, where $E_{k, j}$ is determined by:

$$
E_{k, j}=C_{j}^{\text {min }} \cdot \eta_{j}^{-}\left(t_{k, j}+C_{j}^{\text {min }}\right)-\sum_{p=1}^{k-1} \mathcal{I}_{p, j}
$$

\section{SCHEDULING OVERHEAD}

The necessary schedulability condition valid for any task set and scheduling strategy is

$$
U_{\text {overall }} \leq 1
$$

where $U_{\text {overall }}$ represents the overall resource utilization, and is defined as

$$
U_{\text {overall }}=\sum_{i=1}^{n} \frac{C_{i}^{\max }}{\mathcal{P}_{i}}+U_{R T O S}
$$

In other words, the overall resource utilization is the sum of the average load generated by the tasks executed on that resource and the load generated by the scheduling algorithm.

In Round-Robin scheduling, the RTOS subroutine is executed whenever the scheduler reevaluates the task priorities, i.e when a time slot expires, or when the execution demand of the currently executed task has been completely processed. We call scheduler task, denoted $\tau_{O S}$, the RTOS subroutine executed in order to reassign the task priorities.

The upper bound for the RTOS load, $U_{R T O S}$, is computed as

$$
U_{R T O S}=\sum_{i=1}^{n} \frac{\left[\frac{C_{i}^{\max }}{\theta_{i}-C_{O S}}\right\rceil \cdot C_{O S}}{\mathcal{P}_{i}}
$$

where $C_{O S}$ represents the maximum execution demand of the scheduler task.

Obviously, each task $\tau_{i}$ requires maximum $\left[\frac{C_{i}^{\max }}{\theta_{i}-C_{O S}}\right]$ time slots $\theta_{i}$, in order to complete its execution. Notice that, in every time slot $\theta_{i}, C_{O S}$ units of time are used by the scheduler task. Assuming one execution of $\tau_{O S}$ at the beginning of every time slot, one can compute the RTOS overhead corresponding to the execution of each task, and therefore to determine the total RTOS overhead for the scheduling of the entire task set.

In the following we assume that no scheduling overhead occurs due to switching between different task instances executed in the same time slot. This is not a limitation of the approach, and the response time equations can be easily extended to account also for this aspect.

To capture the time spent during context switches, line 5 in Algorithm 1 is modified as follows:

$$
w_{i}(q)=q \cdot C_{i}^{\max }+\mathcal{I}(q)+\left\lceil\frac{q \cdot C_{i}^{\max }}{\theta_{i}-C_{O S}}\right\rceil \cdot C_{O S}
$$

The value of $K(q)$ in Equation 3 changes to:

$$
K(q)=\left\lceil\frac{q \cdot C_{i}^{\max }}{\theta_{i}-C_{O S}}\right\rceil
$$

In every RR-turn there is one execution of the scheduler task at the beginning of every time slot $\theta_{j}$ only if the execution demand of $\tau_{j}$ at the beginning of $\theta_{j}$ is larger than zero.

The weighting function $\omega_{k, j}$ captures the execution demand of $\tau_{j}$ at the beginning of its time slot in the $k$-th RR-turn.

$$
\omega_{k, j}=\left\{\begin{array}{cc}
0 & E_{k, j}=0 \\
1 & E_{k, j} \neq 0
\end{array}\right.
$$

where $E_{k, j}$ is defined as

$$
E_{k, j}=C_{j}^{\text {max }} \cdot \eta_{j}^{+}\left(t_{k, j}\right)-\sum_{p=1}^{k-1}\left(\mathcal{I}_{p, j}-\omega_{p, j} \cdot C_{O S}\right)
$$

Equation 4 changes to

$$
\mathcal{I}_{k, j}=\omega_{k, j} \cdot C_{O S}+\sum_{r=1}^{R} \operatorname{pad}_{k, j}(r)
$$


The upper-bound arrival function $\eta_{j}^{+}$in Equation 5 must also consider the task activations that eventually arrived during the execution of the scheduler task at the beginning of time slot $\theta_{j}$. The value of $\operatorname{pad}_{k, j}$ is computed as follows:

$$
\begin{aligned}
\operatorname{pad}_{k, j}(r)= & \min \left(l_{k, j}(r), C_{j}^{\text {max }} \cdot \eta_{j}^{+}\left(t_{k, j}+C_{O S}+\right.\right. \\
& \left.\sum_{s=1}^{r-1} \operatorname{pad}_{k, j}(s)\right)-\sum_{p=1}^{k-1}\left(\mathcal{I}_{p, j}-\omega_{p, j} \cdot C_{O S}\right)- \\
& \left.\sum_{s=1}^{r-1} \operatorname{pad}_{k, j}(s)\right)
\end{aligned}
$$

The calculation of the unused time in time slot $\theta_{j}$ at the beginning of $\operatorname{pad}_{k, j}$ changes to

$$
l_{k, j}(r)=\theta_{j}-\omega_{k, j} \cdot C_{O S}-\sum_{s=1}^{r-1} p a d_{k, j}(s)
$$

The time instant at the beginning of $\theta_{j}$ in the $k$-th turn is computed using Equation 7.

The initial value of $\operatorname{pad}_{k, j}$ must also account for the effects of the scheduler task, as follows:

$$
\operatorname{pad}_{k, j}(1)= \begin{cases}\theta_{j}-C_{O S} & E_{k, j} \geq \theta_{j}-C_{O S} \\ E_{k, j} & E_{k, j}<\theta_{j}-C_{O S}\end{cases}
$$

where $E_{k, j}$ is calculated using Equation 20.

Consider again the example presented in Section 3. We carried out the worst-case response time analysis for the defined task set, this time considering the influences of the scheduler tasks. The scheduler task is assumed to have a constant execution demand $C_{O S}=0.2$.

Table 3 shows the influence of $\tau_{O S}$ on the response times of the tasks.

Table 3: The worst-case response times incl. the OS overhead

\begin{tabular}{|l|c|c|c|c|}
\hline Task & $T 1$ & $T 2$ & $T 3$ & $T 4$ \\
\hline \hline w/o $\tau_{O S}$ & 46 & 60 & 31 & 32 \\
w/ $\tau_{O S}$ & 60 & 61.6 & 31.4 & 33 \\
\hline
\end{tabular}

\section{CONCLUSION}

In this paper we addressed the problem of schedulability analysis of tasks under Round-Robin. First, we presented an exact algorithm to compute the worst-case response time of a task, assuming the task can be preempted at any instant. Then, we derived a best-case response time algorithm using a conservative approximation of the best-case scheduling scenario. In the last section we extended the worst-case response time equations to consider the timing overhead due to the scheduler task. The model used for computation uses a 4-function interface to describe the workload arrival. This interface easily allows the analysis of tasks with arbitrary arrival models. Table 4 shows the characteristics of different Round-Robin scheduling analyses. Notice that, the approach presented in this paper supports complex analysis scenarios, and the simple underlying model makes it very intuitive for the analysis of real-time applications.

Table 4: Characteristics of different Round-Robin scheduling analyses

\begin{tabular}{|l|c|c|c|}
\hline & Algorithm 1 & Raha [14] & Migge [12] \\
\hline \hline WCRT & $\sqrt{ }$ & $\sqrt{ }$ & $\sqrt{ }$ \\
BCRT & $\sqrt{ }$ & - & - \\
OS Overhead & $\sqrt{ }$ & - & - \\
Jitter, Burst & $\sqrt{ }$ & - & $\sqrt{ }$ \\
Arbitrary deadlines & $\sqrt{ }$ & - & - \\
Arbitrary arrival models & $\sqrt{ }$ & $\sqrt{ }$ & $\sqrt{ }$ \\
\hline
\end{tabular}

\section{REFERENCES}

[1] R. Budruk, D. Anerson, and T. Stanley. PCI-Express System Architecture. MindShare, Inc., 2003.

[2] FlexRay. http://www.flexray.com/

[3] J. P. Gutierrez, J. J. G. Garcia, and M. G. Harbour. Best-Case Analysis for Improving the Worst-Case Schedulability Test for Distributed Hard RealTime Systems. In Proceedings of the Euromicro Conference on Real-Time Systems (ECRTS), 1998.

[4] S. Heithecker, A. do Carmo Lucas, and R. Ernst. A High-End Real-Time Digital Film Processing Reconfigurable Platform. EURASIP Journal on Embedded Systems, Special Issue on Dynamically Reconfigurable Architectures, 2007:Article ID 85318, 15 Pages, 2007.

[5] K. Jeffay, F. D. Smith, A. Moorthy, and J. Anderson. Proportional Share Scheduling of Operating System Services for Real-Time Applications. In IEEE Real-Time Systems Symposium, Madrid, Spain, 1998.

[6] M. Joseph and P. Pandya. Finding Response Times in a RealTime System. The Computer Journal, 29(5):390-395, 1986.

[7] S. Kanhere and H. Sethu. On the Latency Bound of Pre-Order Deficit Round Robin. In Proceedings of the IEEE Conference on Local Computer Networks, pages 508-517, Tampa, FL, 2002.

[8] H. Kopetz and G. Bauer. The Time-Triggered Architecture. Proceedings of the IEEE, Special Issue on Modeling and Design of Embedded Software, 2001.

[9] J. Lehoczky. Fixed Priority Scheduling of Periodic Task Sets with Arbitrary Deadlines. In Proceedings of the Real-Time Systems Symposium, pages 201-209, 1990.

[10] C. L. Liu and J. W. Layland. Scheduling algorithms for multiprogramming in a hard-real-time environment. Journal of the ACM, 20(1):46-61, 1973.

[11] J. Migge. Real-time scheduling: a trajectory based model. PhD thesis, Nice University, Nancy, France, 1999.

[12] J. Migge, A. Jean-Marie, and N. Navet. Timing analysis of compound scheduling policies: application to Posix1003.1b. Journal of Scheduling, 6(5):457-482, 2003.

[13] S. Mukherjee, D. Saha, M. Saksena, and S. K. Tripathi. A Bandwidth Allocation Scheme for Time Constrained Message Transmission on a Slotted Ring LAN. In IEEE Real-Time Systems Symposium, Raleigh-Durham, NC, 1993.

[14] A. Raha, S. Kamat, and W. Zhao. Guaranteeing End-to-End Deadlines in ATM Networks. In International Conference on Distributed Computing Systems, Vancouver, Canada, 1995.

[15] K. Richter. Compositional Performance Analysis. PhD thesis, Technical University of Braunschweig, 2004.

[16] M. Shreedhar and G. Varghese. Efficient Fair Queueing Using Deficit Round Robin. In SIGCOMM, pages 231-242, Boston, 1995.

[17] B. Sprunt, L. Sha, and J. Lehoczky. Aperiodic Task Scheduling for Hard Real-Time Systems. Journal of Real-Time Systems, 1(1):27-60, 1989.

[18] H. Takagi. Exact analysis of round-robin scheduling of services. IBM J. Res. Dev., 31(4):484-488, 1987.

[19] K. Tindell, A. Burns, and A. Wellings. An Extendible Approach for Analysing Fixed Priority Hard Real-Time Systems. Journal of Real-Time Systems, 6(2):133-152, Mar 1994.

[20] R. Wu and Y.-B. Chen. Analysis of a loop Transmission System with Round-Robin Scheduling of Services. IBM J. Res. Dev., 19(5):486-493, 1975.

[21] R. Yates. High Speed Round Robin Queueing Networks. PhD thesis, MIT, Dept. of Electrical Engineering and Computer Science, 1990. 